$\Rightarrow$ COLORECTAL CANCER

\section{Engineered colons for cancer research}

Experimental animal models for clinically translating basic cancer research are often hindered by applicability limitations. Now, an ex vivo model for studying colorectal cancer (CRC) has been developed to overcome such limitations, involving the recellularization of human colonic tissue with genetically engineered cells containing important mutations for CRC. This system mimicked the pathophysiological progression of CRC and enabled the identification of novel cancer driver genes.

Elucidating the roles of numerous genetic mutations in cells is a crucial but daunting task in understanding cancer. To address this problem, a multidisciplinary collaboration was formed, which included researchers who pioneered the use of the Sleeping Beauty (SB) transposon system to identify cancer driver mutations, and a group who had established a gastrointestinal tract organoid model using human colonic epithelial cells. "We were intrigued by the idea to develop organotypic ex vivo CRC tissues that are tractable and can quickly recapitulate most features of CRC," explains author Huanhuan Joyce Chen.

To achieve this aim, the researchers first obtained normal colon tissues adjacent to tumour sites in patients with CRC. These tissues were decellularized using detergents, preserving the native tissue architecture of the organ. Then, in combination with the SB transposon system, the colon matrix was recellularized with epithelial cells containing mutations in APC (a gene critical for CRC initiation), which resulted in malignant submucosal invasion by multiple adenomas within 6-7 weeks. The adenomas were genetically
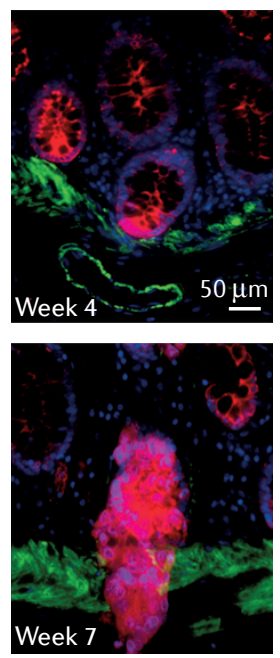

Immunostained images of cytokeratin (red) and fibronectin (green) showing submucosal neoplasia invasion in colonic tissues recellularized with mutant epithelial cells. Nuclei are blue. Courtesy of H.J. Chen profiled to identify SB-mutated genes that cooperate with mutant $A P C$, which revealed 38 candidate invasion driver genes - six of which were novel and might explain how invasion events occur.

"Our study demonstrated the potential of the ex vivo human cancer model to bridge the gap between animal models and clinical diseases," says author Zhubo Wei. "Our model ... will help us better understand the mechanisms of CRC progression at different stages and develop novel therapeutics," he concludes, adding that further engineering could be applied to incorporate vascular networks, the immune system and the gut microbiota. This new engineering methodology might also help generate ex vivo cancer systems for other organs.

Iain Dickson

ORIGINAL ARTICLE Chen, H. J. et al. A

recellularized human colon model identifies cancer driver genes. Nat. Biotechnol. http://dx.doi. org/10.1038/nbt.3586 (2016) 